\title{
Ifosfamide/Mesna/Paclitaxel Regimen
}

National Cancer Institute

\section{Source}

National Cancer Institute. Ifosfamide/Mesna/Paclitaxel Regimen. NCI Thesaurus. Code C160502.

A chemotherapy regimen consisting of ifosfamide, mesna and paclitaxel that can be used in the treatment of uterine, ovarian, fallopian tube, or primary peritoneal cancer. 